\title{
Cross flow water turbines: HARVEST technology
}

\author{
Jean-Luc Achard ${ }^{1, *}$, Favio Dominguez ${ }^{1}$, and Christophe Corre $^{2}$ \\ ${ }^{1}$ LEGI UMR5519, BP53, 38041 Grenoble Cedex 9, France \\ ${ }^{2}$ LMFA UMR5509, Ecole Centrale de Lyon, 36 avenue Guy de Collongue, 69134 Ecully Cedex, France
}

\begin{abstract}
This paper describes all the main features of the hydrokinetic devices developed during the HARVEST program. Such devices, which eliminate the well-known weaknesses of Darrieus turbines, are composed of two counter-rotating twin columns of cross flow water turbines (CFWT) built in a support structure with a twin towers shape. Among other advantages, it is shown that the twin towers geometry facilitates the optimal design of arrays of HARVEST Power Systems. In particular simple 2D models can be used in a first approach to predict power extraction of single-row arrays. Blade Element MomentumReynolds-Averaged Navier-Stokes Simulations (BEM-RANS) models are currently developed to yield a fast and accurate prediction of the power output produced by a row of many power systems. Relevant momentum source terms describing the rotor effects, which are required by this simplified modelling, are defined beforehand from a set of high-fidelity Unsteady Averaged Navier-Stokes Simulations (URANS) simulations for an isolated HARVEST Power System.
\end{abstract}

\section{Introduction}

There are many different concepts of cross flow water turbine (CFWT), originally developed for wind by Darrieus [1]. Some of the key advantages of water CFWTs are: their insensitivity to changes in flow direction; their cylindrical shape allows them to be stacked as part of fences capturing more of the cross sectional area of the current flow than possible with a single diameter horizontal axis turbine; they can directly drive a generator above water level (see Fig. 1) and their rectangular ducts (see Fig. 1c) are much easier to fabricate than ducts of circular sections (annular diffusers) especially when they include slots.

Section 2 reviews the hydraulic operation of a typical CFWT and provides significant parameters. Cross-flow water turbines have also disadvantages that explain their relative rarity in most projects. These disadvantages include: difficulties for self-starting, shaking and lowered power coefficient. The HARVEST program [2], that took place over ten years in Grenoble (France), has sought to bring remedies for these disadvantages. The HARVEST Power Systems (HPSs) are currently commercialized by Hydroquest (France). They are made of an assembly of CFWTs of H-type. Each turbine consists of several vertical straight or V-shaped blades that run along a cylindrical surface and, thanks to their airfoil wing profile, produce a lift force causing the blades to move proportionally faster than the speed of the surrounding water. Turbines are stacked on contra-rotating juxtaposed vertical shafts to

\footnotetext{
* e-mail: Jean-Luc.Achard@legi.grenoble-inp.fr
}

form two columns housed in a twin towers structure composed of a center bow and of two vertical side fairings. Each shaft, which is subjected to turbines torques, is coupled to a generator housed just above the water or on the sea or river floor. The various milestones in the development of the HPSs are indicated in Section 3.

The last step in this development is to predict the power output expected from a specific array. In order to capture significant energy, hydrokinetic turbines need to be built in large arrays within regions such as rivers, man-made channels or tidal straits where the local bathymetry focuses the flow. The layout of these arrays of turbines can significantly change the amount of energy captured from the flow which must be predicted with a high level of certainty to minimize the risks taken by developers and stake holders. Numerical modelling simulations lower the risk and cost for power output prediction, although there remains a need to validate the results against measured data. This paper gives a brief overview of how Computational Fluid Dynamics (CFD) tools are currently used in the last step of this program. The present overview is focused on $2 \mathrm{D}$ analysis, assuming effects in the vertical direction remain negligible.

Section 4.1 gives the general principles of the specific BEM-RANS approach we have developed. Section 4.2 describes how the performance of a single HPS can be accurately computed using a high-fidelity URANS approach; these calculations are necessary to provide momentum sources required by the BEM-RANS calculations to account for the supporting structure effects of the twin towers. Eventually, some results of the HARVEST BEM-RANS model are presented in 
a)

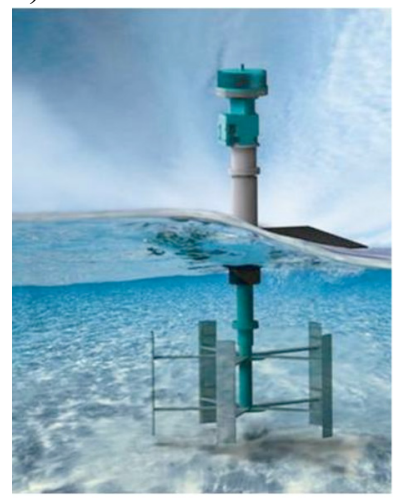

b)

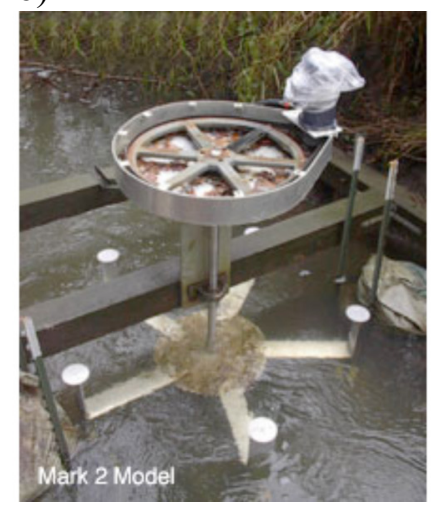

c)

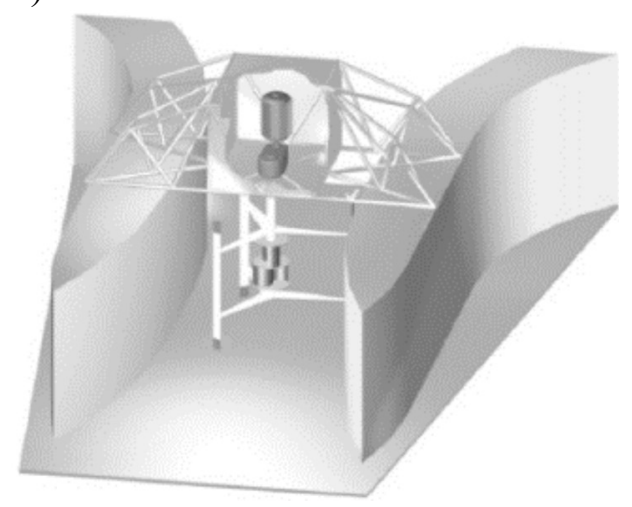

Fig. 1. (a) Go En CFWT, (b) Mark 2 CFWT and (c) FIUBA's diffuser augmented floating CFWT.

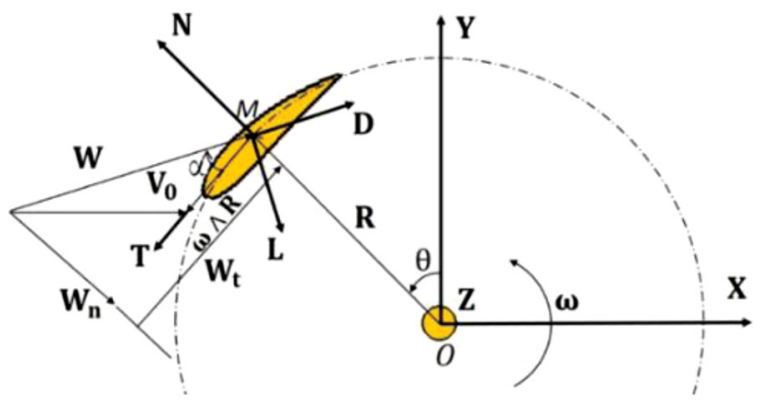

Fig. 2. Transversal cross section of a HARVEST rotor displaying a single blade and the turbine hub. The flow is oriented from left to right.

Section 4.3. The averaged velocity contours around an array of three HPSs located in a uniform channel flow are computed. The variation of the average power coefficient (BEM-RANS prediction) for a single-row array as a function of the lateral spacing $L_{S}$ is also given. Conclusions are drawn and perspectives of future work proposed in Section 5.

\section{A CFWT operation}

Blades of a typical CFWT rotate around the vertical axis with a characteristic rotation vector $\boldsymbol{\omega}$. The local flow velocity can be expressed either in a fixed reference frame or in a moving reference frame, attached to the rotating blade. If $V_{0}$ denotes the absolute local flow velocity upstream of the turbine blade, the corresponding local velocity $\mathbf{W}$ of the fluid relative to the moving blade is given by:

$$
\mathbf{W}=V_{0}-\mathbf{\omega} \wedge \mathbf{R},
$$

where $\mathbf{R}$ denotes the position vector of the blade center (mid-chord) in the 2D cylindrical coordinates system $(0, \theta$, $\mathbf{R})$ depicted in Figure 2. The angular position $\theta$ of the blade in this reference frame is equal to 0 when the blade center lies on the $Y$-axis and increases when the blade is rotating counterclockwise. The angle of attack $\alpha$ is defined as the angle between the local relative velocity $\mathbf{W}$ upstream of the blade and the blade chord line:

$$
\alpha=\arctan \left(\frac{\mathbf{W} \times n}{\mathbf{W} \times t}\right) .
$$

Three distinct reference frames can be distinguished in Figure 2: (i) the absolute reference frame, where Cartesian coordinates $X, Y$ or cylindrical coordinates can be used; (ii) the reference frame relative to the flow, with axes respectively aligned with the direction of the relative velocity and orthogonal to this direction, in which the hydrodynamic force applied by the fluid to the blade is decomposed into the $\operatorname{drag} D$ and lift $L$ components; (iii) the reference frame relative to the blade, with axes respectively tangential and normal to the turbine rotation center, in which the hydrodynamic force is decomposed into a normal $F_{n}$ and tangential $F_{t}$ components. The hydraulic operation of the turbine can be characterized by the power output $P$ of each rotor per unit height, computed from the rotor torque $C$ and the angular velocity as $P=C \boldsymbol{\omega}$. The instantaneous value of the torque $C$ is computed for each angular position $\theta$ of the blade from the tangential force component $F_{t}$ which yields an instantaneous value for the power output $P$. It is customary to express the force components and also the power output in non-dimensional form. The drag and lift coefficients and the normal and tangential force coefficients of a turbine blade are defined as:

$$
\begin{aligned}
C_{D} & =\frac{F_{d}}{0.5 \rho c W^{2}}, \quad C_{L}=\frac{F_{l}}{0.5 \rho c W^{2}}, \quad C_{T} \\
& =\frac{F_{t}}{0.5 \rho c W^{2}}, \quad C_{N}=\frac{F_{n}}{0.5 \rho c W^{2}},
\end{aligned}
$$

where $c$ denotes the blade chord and $W$ is the magnitude of the reference relative velocity $W$ upstream of the blade. These force coefficients depend on the angle of attack $\alpha$ and the Reynolds number but the Reynolds effect can be neglected in the present study because the typical value of the Reynolds number is high enough (more than two million based on inflow velocity and rotor diameter) to ensure fully turbulent flow. The turbine power coefficient per unit height is defined as:

$$
C_{P}=\frac{P}{0.5 \rho V_{r e f}^{3} D},
$$


a)

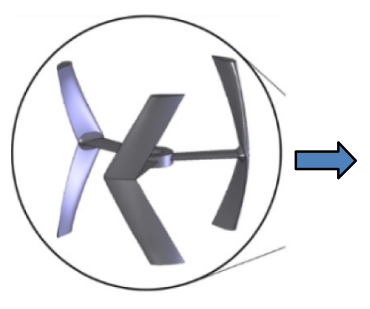

b)

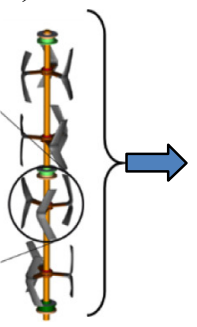

c)

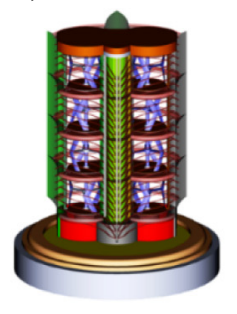

d)

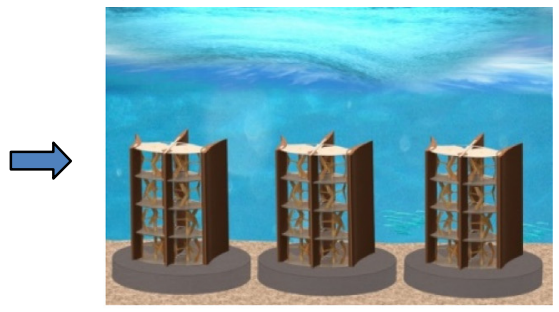

Fig. 3. Four steps (a) a new CWFT, (b) a column of turbines, (c) a HARVEST Power System (HPS), (d) an array of HPS.

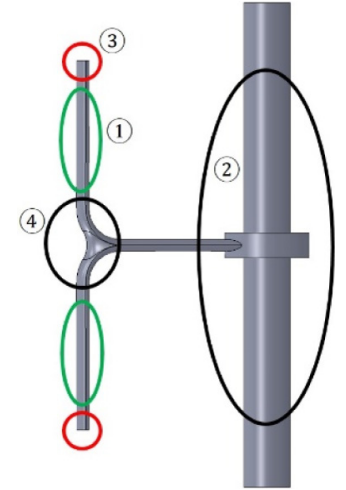

Fig. 4. Physical phenomena conditioning performance.

where the turbine diameter $D$ is used as characteristic length; the reference velocity $V_{r e f}$ is an absolute velocity which can be for instance the upstream farfield velocity $V_{0}$ in the case of a uniform upstream farfield flow. The reference velocity is also used to define a non-dimensional rotational speed, the tip speed ratio $(T S R) \lambda$ such that:

$$
\lambda=\frac{\omega R}{V_{0}} .
$$

\section{The HARVEST program: a new concept}

The HARVEST program has been developed following four steps described in Figure 3, each step incorporating the results of the previous step.

The first step aimed to define an optimized CFWT module. To improve performance, the following four sources of viscous dissipation have to be reduced by an appropriate design as illustrated in Figure 4. Two viscous vortex structures are 2D: (1) Airfoil Dynamic Stall, (2) Von Karman Vortices and the other two are 3D: (3) Blade Tip Induced Vortices, (4) Wing-Wing Junction Horseshoe Vortices. The hydrodynamic behavior of various models has been analyzed both experimentally and numerically. On the one hand a hydrodynamic channel has been equipped with a measurement platform which has provided instantaneous and averaged measurements of $2 \mathrm{D}$ thrusts as well as hydrodynamic torques. Velocity components have also been obtained by using a PIV technique.

On the other hand various CFD codes based on 2D and 3D formulations of the Unsteady Reynolds-Averaged Navier-Stokes (URANS) equations and employing the

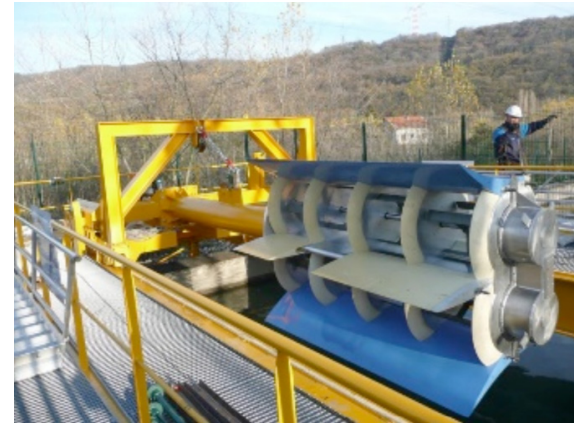

Fig. 5. A prototype HPS before being immersed.

$k-\omega S S T$ turbulence closure model have been applied to the analysis of the CFWT module. Two or three straight bladed H-type CFWT modules result from these studies with specifically designed Arm-Blade Junctions and Blade Tips. Blades can be straight or V-shaped and attached to the central shaft with one or two sets of horizontal arms.

In a second step, the optimized turbines are stacked on a single rotating axis to form a column. Such a structure offers several advantages: (i) All turbines sharing the same rotating vertical shaft may also share a common generator which can be tuned, so that the rotational speed of the column systematically makes each turbine work at the optimum level of its power curve. (ii) The possibility of combining several identical turbines according to each site enhances modularity and optimizes productivity. (iii) As turbine positions can be staggered, the torque delivered to the column, and then to the generator, can be smoothed. (iv) The modest size of the turbines (say $3-5 \mathrm{~m}$ for sea applications and 1-3 $\mathrm{m}$ for river applications) allows mass production as well as an easy installation and maintenance.

In a third step, two counter-rotating twin columns stacked with turbines are built into a supporting structure composed of a center bow and horizontal plates between each stage. In this way (i) lift forces and vibrations on each column are cancelled. Moreover, the supporting structure is equipped with vertical non-symmetrical lateral fairings to form a diffuser; such fairings (ii) create over speed in the drive areas of the turbines augmenting the power extracted for turbines of given size and (iii) contribute to the opposing support structure and foundations. Furthermore (iv) horizontal plates reduce tip losses and improve efficiency. A two meters high HPS shown in Figure 5 has been manufactured and successfully tested in a feed channel of a hydroelectric plant near Grenoble (France). 

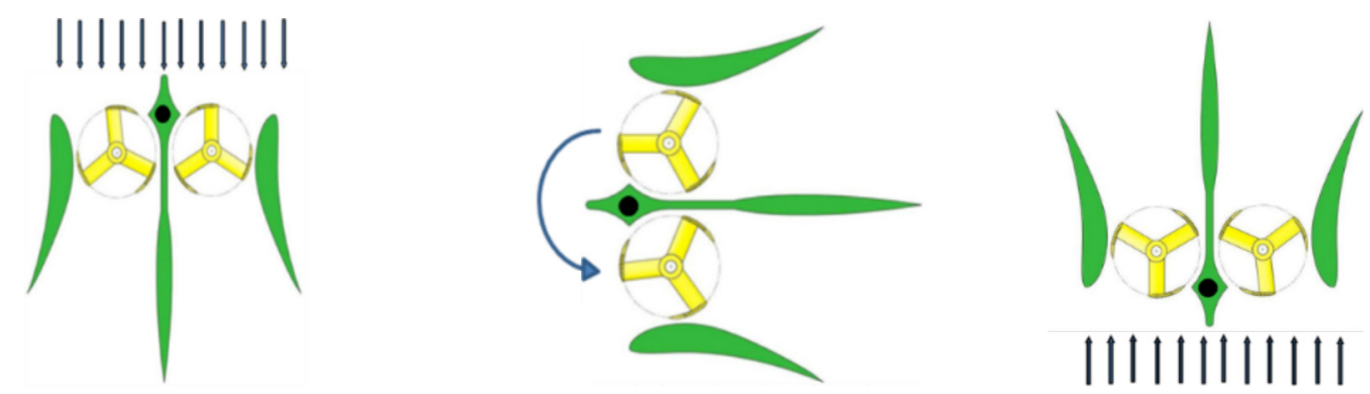

Fig. 6. Auto-orientation of the HPS facing the current, with a rotation of about $180^{\circ}$ during the change of tide.

Finally (v) introducing a pylon across the bow leaves the twin towers (which are free to rotate) move by themselves to face the current and opposes the drag force to prevent the HPS to overturn (Fig. 6).

In a fourth step, for a single model of the basic HPS, it is possible to make the most of a wide variety of topology of potential sites. To rationally exploit the energy characteristics of the incident current, the best distribution of HPSs across a river is sought. This step keeps on being performed at LEGI in a current cooperation with Hydroquest which extends the HARVEST program.

\section{Development of the BEM-RANS model}

\subsection{General design principles}

The BEM-RANS approach solves the steady RANS equations in a computational domain where the rotor blades have been suppressed and replaced with computational cells discretizing the surface swept by the rotor blades over a period of revolution. Figure 7 displays a schematic view of this discretized swept surface used for the rotor description. The RANS equations solved by the BEMRANS approach are similar to the URANS system; the continuity equation remains unchanged while the momentum equation reads now:

$$
\frac{\partial \bar{U}_{i} \bar{U}_{j}}{\partial x_{j}}=-\frac{1}{\rho} \frac{\partial \bar{P}}{\partial x_{i}}+\frac{\partial}{\partial x_{j}}\left[v\left(\frac{\partial \bar{U}_{i}}{\partial x_{j}}+\frac{\partial \bar{U}_{j}}{\partial x_{i}}\right)\right]-\frac{\partial \overline{u_{i}^{\prime} u_{j}^{\prime}}}{\partial x_{j}}+S_{i},
$$

where the source term $\mathbf{S}$ is active in each cell of the virtual rotor surface. It must be defined in such a way it accounts for the time-averaged (over a period of rotation for the rotor) hydrodynamic effects of the rotating blades. The instantaneous force applied on the flow by the blade element is expressed in the fixed system coordinate $(0, X$, $Y)$ by projecting $\left(-F_{l},-F_{d}\right)$ or $\left(-F_{n},-F_{t}\right)$ onto the $X$ and $Y$-axes. These instantaneous values must be timeaveraged over one period of revolution before being inserted into (6). The $X$ and $Y$ components of the momentum source term $S^{(k)}$ introduced in the $k$ th cell of the virtual rotor, identified by the angular position $\theta^{(k)}$, are computed as:

$$
S_{X, Y}^{(k)}=-\frac{N \times F_{X, Y}^{(k)}}{A}
$$

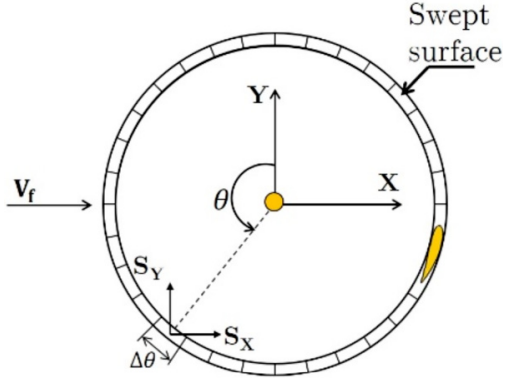

Fig. 7. Schematic view of the discretized swept surface used in the BEM-RANS model. A value of the BEM source term $\mathbf{S}$ is computed in each grid cell.

where $N$ is the number of rotor blades $(N=3$ here) and $A$ is the area of the surface swept by the blades. The components $F_{X, Y}^{(k)}$ of the force applied in the $k$ th cell of the virtual rotor can be deduced from the lift and drag computed in this same cell or from the normal and tangential force components using simple geometric transformations, namely rotations of angle $\theta^{(k)}$ and $\alpha^{(k)}$ where the angle of attack $\alpha^{(k)}$ is associated with the $k$ th cell of the virtual rotor. The lift and drag can be computed using the non-dimensional force coefficients introduced in (3):

$$
F_{l, d}^{(k)}=C_{L, D}\left(\alpha^{(k)}\right) \frac{1}{2} \rho\left[W^{(k)}\right]^{2} c .
$$

Since $\alpha^{(k)}$ can be computed from $W^{(k)}$ using (2), the source term (7) is entirely defined once the local reference velocity $W^{(k)}$ is itself defined and the non-dimensional lift and drag coefficients $C_{D}, C_{L}$ for the rotor blade are available. A customary practice when applying the BEMRANS approach is to use polar plots for $C_{D}, C_{L}$ which are already available for the blade profile used in the turbine design. Such a straightforward approach cannot be applied in the present case because of the ducted design of the turbine which introduces confinement effect not properly taken into account in polar plots obtained from free-flow experiments performed on the blade profile. Hence, preliminary URANS simulations are needed to build relevant tables of values for $C_{D}, C_{L}$. Once these tables obtained and stored, the BEM-RANS model calculates the flow through a CFWT with the moving rotor grid replaced with a fixed grid of the surface swept by the rotor. System (6) is solved with $\mathbf{S}$ computed using $W^{(k)}$ extracted from the current velocity field. The solution of 
(6) yields a new velocity field leading to updated values of the source term $\mathbf{S}$. This process is iterated until a steady state is reached with steady force components, thus the expected converged value for the power coefficient of interest.

The cost reduction of the BEM-RANS model with respect to the baseline URANS approach for predicting the power output of a twin towers array results from two factors: (i) a reduced number of computational cells, since the highly refined mesh around the rotor blades is replaced with a much coarser 1D mesh of the surface swept by the blades, and (ii) the replacement of an unsteady simulation with a steady simulation. However the local velocity relative to the blade $W^{(k)}$ must be computed from a local absolute velocity $V^{(k)}$ relative to cell $k$ of the virtual rotor and from the rotational speed of the turbine $\boldsymbol{\omega}$ using (1). The URANS model should then be applied for several values of $\boldsymbol{\omega}$ or TSR $\lambda$ until the optimal value of $\lambda$ is reached. The cost of the URANS model would thus remain rather high because of the persistent need for a parametric study to determine the optimal set of TSR values for all the turbines. To overcome this difficulty, a methodology to derive a BEM-RANS model for turbines working at their optimal TSR is described in [3]. It leads to the proper calibration of two universal curves used to feed the BEMRANS model at the optimal TSR. For the upstream part of the virtual BEM-RANS rotor, the coefficients $C_{D}, C_{L}$ defined for a relative reference velocity are expressed in terms of the local angle of attack while for the remaining downstream part of the virtual BEM-RANS rotor the coefficients $C_{X}, C_{Y}$, defined with the flow rate velocity through the turbine, are expressed in terms of the angular position.

\subsection{An isolated HPS in a channel}

The above calibration is achieved through an extensive analysis of simulations based on URANS equations governing the unsteady turbulent flow performed for an isolated twin towers in a channel with various blockage ratios and inflow velocity conditions, non-necessarily uniform. A single HPS is placed inside a rectangular channel and aligned with the incoming flow (horizontal) direction (see Fig. 8). The ratio between the turbine width $L_{T}$ (the distance between the trailing edge of the fairings) and the channel width $W_{C}$ defines the blockage ratio $\varphi$ of the configuration. It has already been emphasized that the HARVEST CWFTs work at an optimal TSR value thanks to its regulation system. Consequently, the prediction of the power coefficient $C_{P}$ requires a series of numerical simulations performed for a fixed value of the TSR parameter $\lambda$ in order to identify the power output corresponding to the a priori unknown optimal value $\lambda^{*}$ of the TSR. An example of computed quantity of interest $C_{P}$ is plotted in Figure 9 for $V_{0}=2.25 \mathrm{~m} / \mathrm{s}$, $\varphi=0.29$ and several test values of the tip speed ratio $\lambda$ for the turbine rotor: the power coefficient of the isolated turbine is found to reach a maximum value $C_{P \max }=1.059$ for the optimal value of $T S R \lambda^{*}=2.3$. The single BEMRANS calculation yields an optimal value of the power coefficient which is very close to the optimal reference

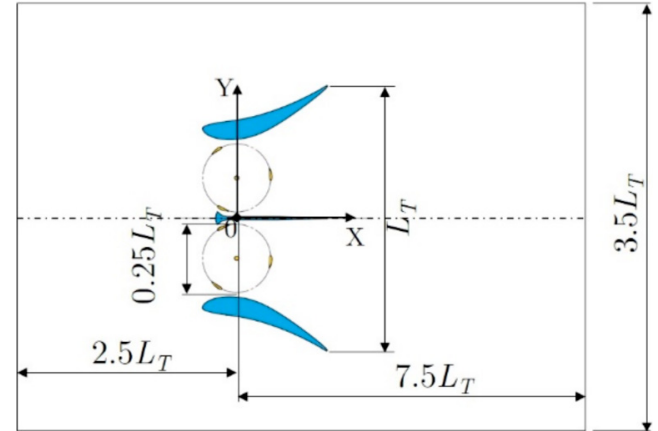

Fig. 8. Isolated HPS in a channel. Overview of the $2 \mathrm{D}$ computational domain. The computational mesh of the sole rotor region contains 110,000 cells while the remainder of the domain, outside the rotor region, contains about 135,000 cells resulting in a total number of cells approximately equal to 245,000 .

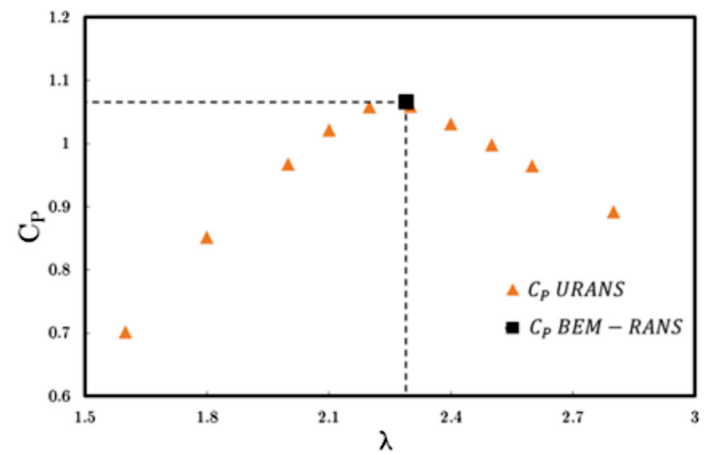

Fig. 9. Power coefficient of an isolated HPS in a channel $(\varphi=0.29$ and $\left.V_{0}=2.25 \mathrm{~m} / \mathrm{s}\right)$. Numerical prediction by the URANS model from a set of computations performed for various $\lambda$ compared to the power coefficient of a single steady BEM-RANS calculation.

value obtained from a series of expensive URANS simulation. Note the considered flow conditions (inflow velocity and blockage ratio) were not part of the extensive database used to calibrate the expressions of the hydrodynamic coefficients $C_{D}, C_{L}$ or $C_{X}, C_{Y}$ used in the BEM-RANS model which makes this flow problem a convincing validation of the BEM-RANS model.

\subsection{Exploitation of the BEM-RANS model}

The validated BEM-RANS model is now applied to the analysis of the power output produced by a single row of $n$ HPSs devices aligned across a channel of fixed width $W_{C}=150 \mathrm{~m}$. The spacing $L_{S}$ between each device expressed in dimensionless form becomes $L_{S}^{*}=L_{S} / L_{T}$ with $L_{S}^{*} \geq 1$. The row is supposed to be symmetric with respect to the channel center and the distance $L_{B}$ between the first and last HPS of the row and the closest channel bank is equal in dimensionless form to $L_{B}^{*}=L_{B} / L_{T}$ with $L_{B}^{*} \geq 1$. It is straightforward to establish the relationship: $2 L_{B}^{*}+(n-1) L_{S}^{*}+n=n / \varphi$. Consequently, the power produced by the row of turbines depends on three geometrical parameters only: the numbers $n$ of turbines, the lateral spacing between turbines $L_{S}^{*}$ and the blockage ratio $\varphi$. For the present given channel width and given 


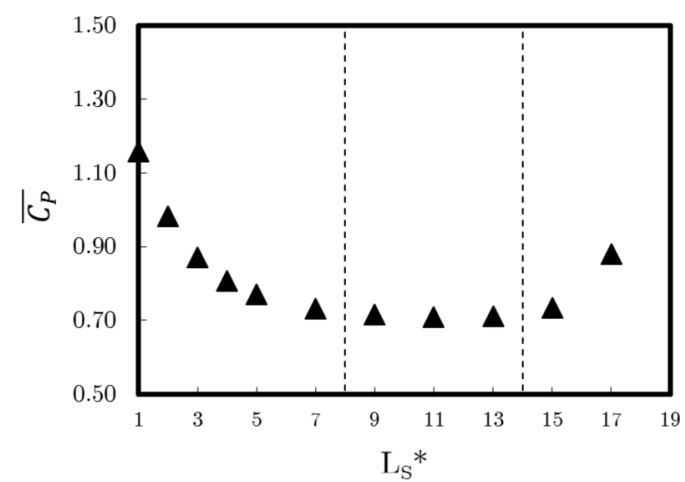

Fig. 10. Variation of the average power coefficient (BEM-RANS prediction) for a single-row array of HPSs with $n=3$ and $\varphi=0.08$ as a function of the lateral spacing $L_{s}^{*}$.

turbine width, the parameters $n$ and $\varphi$ are such that $n /$ $\varphi=37.5$. Moreover, the upstream velocity $V_{0}$ is supposed fixed. The BEM-RANS model is applied to assess the variation of the averaged power coefficient of the row $\left(\overline{C_{P}}=\left(\sum_{i=1}^{n}\left(C_{P}\right)_{i}\right) / n\right)$ with the blockage ratio or the lateral spacing.

Two examples will be now given for the test-problem of the three HPSs row. For a given blockage ratio of $\varphi=0.08$ and a velocity $V_{0}=2.25 \mathrm{~m} / \mathrm{s}$. $C_{P}$ is plotted as a function of $L_{S}^{*}$ in Figure 10. The power coefficient is maximum when $L_{S}^{*}$ is equal to its minimum value $\left(L_{S}^{*}=1\right.$ in the present analysis) because of the beneficial flow acceleration produced by the ducted turbines when close to one another. Next, the value of $C_{P}$ decreases when $L_{S}^{*}$ increases because the flow acceleration is reduced with the turbines increasingly apart. Eventually, the power coefficient increases again because of the flow acceleration produced by the outer ducted turbines getting close to the side boundaries of the domain, corresponding to the channel banks.
The flow configuration corresponding to $\varphi=0.13$, $V_{0}=2.10 \mathrm{~m} / \mathrm{s}$ and $L_{S}^{*}=1$ is computed using both BEMRANS and the URANS approach, this latter necessarily including a parametric study on the TSR values to determine their optimal combination which eventually makes the URANS approach more computationally expensive than BEM-RANS by orders of magnitude. Figure 11 illustrates the very good qualitative agreement between the BEM-RANS and the averaged URANS velocity fields. It can also be shown that the predicted value for $C_{P}$ using BEM-RANS compares again very well with the URANS reference result. The predicted BEMRANS value is equal to $\overline{C_{P}}=1.21$ a difference of $+2.5 \%$ only with respect to the reference URANS calculation. The computational cost of the numerical prediction is divided by a factor larger than 300 when using the BEM-RANS model instead of the high-fidelity URANS approach.

\section{Conclusion}

It has been shown that the HARVEST Power Systems (HPSs) developed via the HARVEST program, the key steps of which were listed, remedy to all the well-known weaknesses of cross flow water turbines (CFWTs). The HPSs turbo machines are composed of optimized CFWTs stacked along two counter-rotating twin columns housed in a support structure composed of a center bow, two vertical side fairings and horizontal plates between each stage. The resulting vertical HPS composed of twin towers have a global geometry inducing a careening effect in the space between them and are particularly suitable for use in clusters. As the last step of the HARVEST program, a BEM-RANS model has been developed to yield a fast and accurate prediction of the power output produced by a HPSs row, while a similar analysis is definitely out of reach for a high-fidelity URANS strategy. The BEM-RANS
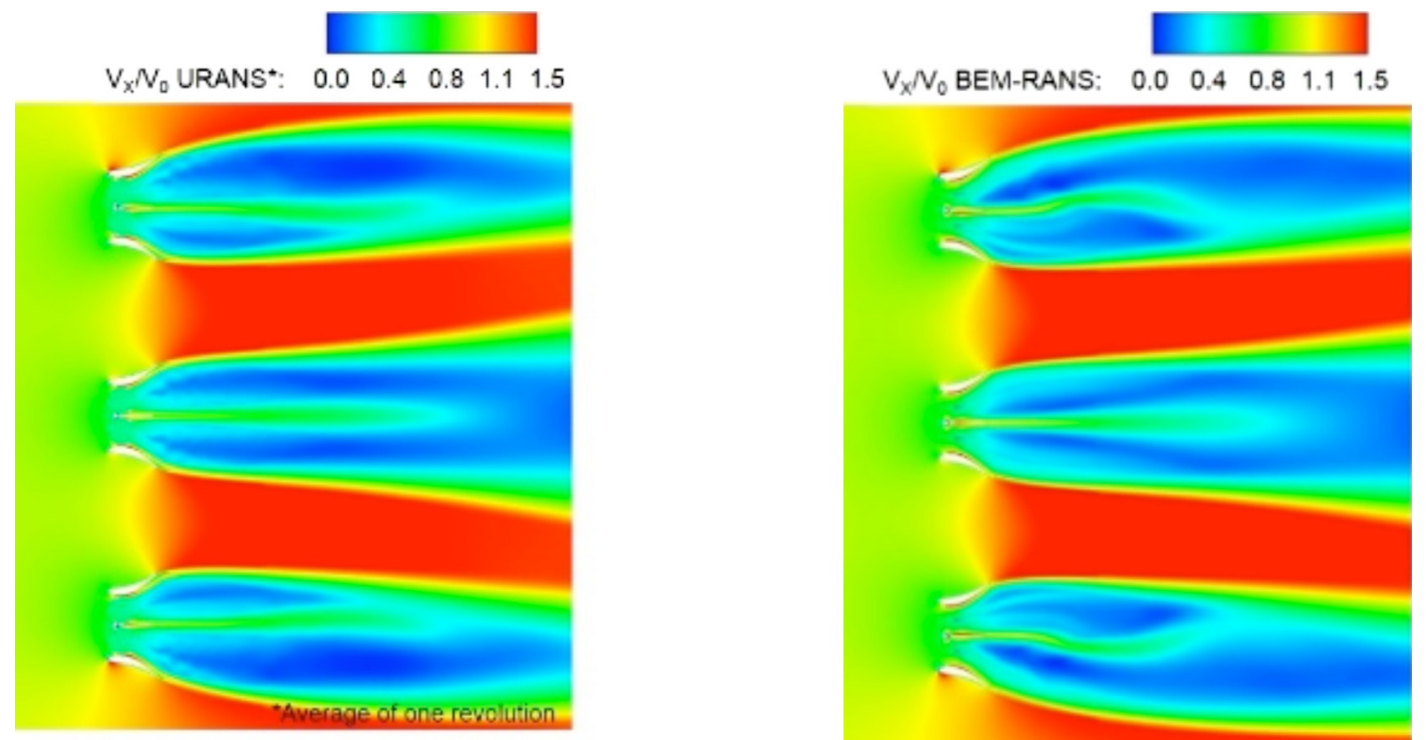

Fig. 11. Row of three HPSs in a channel. Left: averaged (over a period of rotation) velocity contours computed using the reference URANS approach. Right: steady velocity contours computed using the BEM-RANS approach. 
model is currently being improved in order to be applied to multi-row turbine arrays where wake effects will be significant.

\section{References}

1. G.J.M. Darrieus, Turbine having its rotating shaft transverse to the flow of the current, U.S. Patent 1,835,018, December 8, 1931 (1931)
2. J.-L. Achard, D. Imbault, A. Tourabi, Turbine engine with transverse-flow hydraulic turbine having reduced total lift force, U.S. Patent 8,827,631 B2, September 9 (2014)

3. F. Dominguez, J.-L. Achard, J.M. Zanette, C. Corre, Fast power output prediction for a single row of ducted cross-flow water turbines using a BEM-RANS approach, Renew. Energy 89, 658 (2016)

Cite this article as: Jean-Luc Achard, Favio Dominguez, Christophe Corre, Cross flow water turbines: HARVEST technology, Renew. Energy Environ. Sustain. 1, 38 (2016) 